Managing the severely impaired arm after stroke: a mixed-methods study with qualitative emphasis

Running head: Managing the severely impaired arm after stroke

Article category: Original research

Stefan Tino Kulnik ${ }^{1}$, Sushmita Mohapatra ${ }^{2}$, Sara Gawned ${ }^{3}$, Fiona Jones ${ }^{1,4}$

${ }^{1}$ Faculty of Health, Social Care and Education, Kingston University and St George's,

University of London, London, United Kingdom

${ }^{2}$ Therapy Services, King's College Hospital NHS Foundation Trust, London, United

Kingdom

${ }^{3}$ Therapies Department, St George's University Hospitals NHS Foundation Trust,

London, United Kingdom

${ }^{4}$ Bridges Self-Management Limited, London, United Kingdom

Corresponding author:

Dr. Stefan Tino Kulnik

Faculty of Health, Social Care and Education

Kingston University and St George's, University of London

6th Floor Hunter Wing (Desk 15, Bay 19)

St George's Campus

Cranmer Terrace

London SW17 ORE

United Kingdom

Tel +44(0)2087252266

Email s.t.kulnik@sgul.kingston.ac.uk 
Acknowledgements: This research was funded through the First Grant Scheme (number RSCH198F) provided by Kingston University, London, UK. The authors thank study advisors Shani Shamah and Kennedy Amo, and the stroke survivors who participated in the study.

Declaration of interest: FJ is founder and CEO of non-profit organization Bridges SelfManagement Limited. SK has previously worked for Bridges Self-Management Limited. SM and SG have no disclosures. 


\title{
Managing the severely impaired arm after stroke: a mixed-methods study with qualitative emphasis
}

\author{
Abstract
}

PURPOSE: There is a paucity of research into self-management strategies employed by stroke survivors outside of formal rehabilitation. This study aimed to explore stroke survivors' experiences of, and strategies for self-managing the severely impaired upper limb.

MATERIALS AND METHODS: English-speaking stroke survivors whose upper limb had been non-functional at 3 months post-stroke took part in interviews $(n=16)$, a focus group $(n=6)$ and a survey $(n=20)$. Quantitative data were analysed descriptively. Qualitative data were analysed through thematic framework analysis using NVivo(C software.

RESULTS: Participants' median (range) age group was $50-59$ years $(30-39,70+)$. Median (range) time post-stroke was 39 months ( 4 months, 46 years). The overarching theme across the data was 'choosing and expanding'. Participants enacted various professionally driven management strategies for their affected arm, and then chose to continue with what worked for them. Strategies were expanded through self-discovery and interaction with peers. There was sadness and frustration at the loss of the arm, but also satisfaction and self-confidence when progress was being made. Participants described an attitude of not giving up and remaining hopeful.

CONCLUSIONS: These findings extend the current evidence base and can be used to inform a novel stroke self-management intervention for the severely impaired upper limb.

Keywords: self-management, rehabilitation, upper extremity, upper limb 


\section{Introduction}

The annual incidence of stroke in the United Kingdom (UK) exceeds 100,000 [1,2]. Arm weakness occurs in $77 \%$ of stroke survivors [3] and is a major cause of functional disability $[4,5]$. Arm recovery after stroke is therefore considered a research priority [6], and a large body of research into rehabilitation interventions for the upper limb after stroke has accumulated over the past two decades. However, in a recent Cochrane overview of systematic reviews, evidence from 503 trials failed to yield high quality practice recommendations [7]. Practice recommendations are supported by moderate quality evidence at best, and mainly relate to mild or moderate arm weakness and researcher- and clinician-led interventions. The impact of a severely impaired arm on stroke survivors' body image and sense of self is largely unknown and often neglected [8].

Few studies have explored arm recovery from the perspective of stroke survivors. A study by Barker and Brauer from 2005 [9] is the only qualitative research known to the authors. In a sample of 19 Australian chronic stroke survivors, 9 of whom had a nonfunctional upper limb, this study described key themes of 'getting going and keeping going with exercise', finding out how to keep moving ahead' and 'hanging in there'. Losing hope was understood as bad recovery, and participants believed recovery only came to an end when the stroke survivor 'gave up' [9]. Barker and colleagues subsequently conducted a postal survey of 220 Australian chronic stroke survivors, to identify self-reported factors associated with upper limb recovery. Twenty-three percent of respondents reported no upper limb recovery, and $70 \%$ reported ' $50 \%$ or less' upper limb recovery. Factors associated with better perception of recovery were 
hope, confidence, a sense of responsibility for driving one's own recovery, and having the use of the arm in everyday tasks [10].

The work by Barker and colleagues, although presenting a limited body of evidence, resonates strongly with tenets of self-management. Self-management is commonly defined as an individual's ability to manage the symptoms, treatment, physical and psychosocial consequences and lifestyle changes inherent in living with a certain condition [A]. Generic stroke self-management interventions have shown promise, with a recent Cochrane systematic review of 14 trials demonstrating effect sizes (standardized mean difference) on quality of life and self-efficacy of 0.35 (95\% confidence interval 0.05 to 0.62 ) and 0.33 ( $95 \%$ confidence interval 0.04 to 0.61 ), respectively [11]. However, to our knowledge self-management interventions that specifically address the severely impaired arm have not been developed to date. Instead, previous interventions for the severely impaired arm have often focused on 'hands-on' therapy, which can be impeded by scarce rehabilitation resources. There is therefore an untapped opportunity to draw on the experiences of stroke survivors, to discover what strategies they themselves find most useful and relevant for managing the severely impaired arm after stroke, so that these experience-based insights may be put to practical use in a tailored self-management intervention. Importantly, newly developed self-management interventions need to take account of the respective healthcare context [27]. An exploration of current views and experiences of stroke survivors in the UK is therefore warranted.

This study aimed to explore stroke survivors' experiences of, and strategies for, managing the severely impaired arm; and to describe how individuals envisage a selfmanagement intervention for the severely impaired arm. 


\section{Materials and methods}

The study adopted a mixed-methods design with qualitative emphasis [12]. A descriptive phenomenological approach was taken. Semi-structured interviews, a focus group and a survey were used to collect data. This approach offered study participants alternatives for convenience and inclusiveness, for example with respect to mobility restrictions and communication difficulties. The main consideration for including a survey was to offer an option to participants who may not be willing or motivated to take part in a research interview or focus group, for example because they prefer to participate in private or in their own time. Adult (18+ years) stroke survivors who had had severe arm impairment (defined as non-functional upper limb) at 3 months post-stroke were recruited. There was no limit on the time since stroke onset. Recruitment was purposive, to represent different characteristics (time from stroke onset; degree of recovery; flaccidity versus hypertonicity/spasticity). Those who lacked capacity to consent or did not speak English were excluded. Recruitment took place in the Southeast of the UK from February to June 2017. Study invitations (including contact details for the research team and the online survey link) were circulated via relevant regional voluntary sector organisations and stroke networks. Given the study resources and timeline, an estimated sample size of 20 interview and focus group participants and 50 survey respondents was considered achievable. The study was approved by the university research ethics committee (reference FREC 2017-01-013). Interview and focus group participants gave written informed consent. Survey respondents' consent was implied in completing the questionnaire. 


\section{Interviews}

Interviews were conducted by the first author who is experienced in interviewing people with stroke. Participants had the choice of giving an interview in person, over the telephone, or via online video link. A semi-structured interview schedule was used to elicit in-depth accounts of interviewees' experiences of living and managing with their severely impaired upper limb following stroke. The interview schedule was piloted with study advisors ( 2 stroke survivors with personal experience of severe arm impairment) and is available in online supplemental file 1. Interviews were audiorecorded. The interviewer supported participants with cognitive and communication difficulties, through maintaining a slow interview pace; giving interviewees time to formulate answers without interjecting or pre-empting their replies; incorporating non-verbal and written communication; and repeating back what the interviewee had expressed. Concurrent researcher notes were added to the data corpus.

\section{Focus group}

The focus group was held at a community venue, audio-recorded, and facilitated by the first author, using the same questions as in the interview schedule for individual interviews. One of the co-authors (SG) co-facilitated, observing the dynamics and interactions during the group and taking concurrent notes.

\section{Survey}

An online survey questionnaire was designed and piloted by study advisors. Participants could opt to receive a paper version of the questionnaire with pre-paid 
return envelope and telephone assistance from the research team. The survey questionnaire consisted of 25 items with multiple choice and free text answer options. Items covered respondents' personal and stroke characteristics, experiences of living with and managing the severely impaired arm, and views on content and format of a self-management intervention. As quantitative measures of upper limb function, the questionnaire included the ABILHAND Manual Ability Measure [13], and an adapted version of the Arm Activity Measure (ArmA) [14]. A copy of the questionnaire is available in online supplemental file 2 .

\section{Analysis}

Participant characteristics were summarised using descriptive statistics. Interview and focus group recordings were professionally transcribed verbatim. The first author reread transcripts and listened to interview recordings, correcting any transcription errors and removing person-identifiable information. Transcripts and survey responses were uploaded to NVivo (C) software (QSR International, 2017) for coding. Framework analysis [15] was used to code content according to the categories/themes of the interview schedule, including an open coding category for additional emerging themes. Analysis was iterative and ongoing, with analysis of earlier interviews informing later interviews. A preliminary data analysis was presented and discussed in a peer review meeting with study advisors and collaborators, generating a revised analytic framework and refinement of the interpretation of findings. A finalised textual summary and graphic representation of the data analysis was agreed by all study advisors and collaborators. Study reporting followed established quality criteria [16]. 


\section{Researcher reflexivity}

The research team enacted researcher reflexivity by acknowledging their preunderstandings and by incorporating opportunities to reflect on these as data were collected and analysed. The first author took concurrent and retrospective field notes, which included reflective entries. Debriefing sessions and meetings with other members of the research team (including study advisors) provided a forum for open discussion, challenges, alternative views, and ultimately refinement of the analysis.

It is acknowledged that the study was conceived and designed according to theoretical assumptions of the self-management approach that has been developed and researched by the senior author over the past 15 years [27]. Within this approach, in-depth understanding of the stroke survivor's experience constitutes a key principle. Central to this is the question of what stroke survivors themselves have found helpful, based on their lived experience. This information may then serve as a point of departure for designing a selfmanagement intervention for this particular context.

\section{Results}

Sixteen stroke survivors participated in individual interviews, 6 in the focus group, and 20 in the survey. Eleven interviews were conducted face-to-face, and 5 over the telephone, with median (range) interview duration of $56 \mathrm{~min}(35,82)$. The focus group duration was 2 hours. Participant characteristics are given in table 1. 
Findings are presented in two sections, which correspond to the study aims. The first section addresses stroke survivors' experiences of, and strategies for managing the affected arm; and the second section describes how stroke survivors envisage a selfmanagement intervention for the severely impaired arm.

\section{Experiences and strategies for managing the severely impaired arm}

The overarching theme that encapsulated stroke survivors' experiences of managing the severely impaired arm was choosing and expanding, comprising three underpinning subthemes enacting professionally driven advice, investing in recovery and choosing a positive mind-set (figure 1). In the following, themes are described in detail and supported with participants' anonymised direct quotes. The three sub-themes are described first. These sub-themes are conceptualized as notable and prominent aspects of participants' experience, which are situated within and at the same time feed into the overarching theme of choosing and expanding.

\section{FIGURE 1 ABOUT HERE}

Enacting professionally driven advice

This sub-theme describes the unidirectional process in which professionals give advice and guidance, especially during the first weeks and months after stroke, and this is enacted by stroke survivors.

Each participant described a multitude of recommended strategies for managing their stroke-affected arm, including stretches, massage and exercises; involving the arm in every activity where possible; using devices such as aids, splints, braces, electric 
stimulation devices, exercise equipment and gadgets; taking medication and having botulinum toxin injections for spasticity and pain; and caring for the arm (e.g. hygiene measures, specific positioning, etc.). Much of this activity was driven by advice and guidance from rehabilitation professionals, especially in the early weeks and months after stroke. This was a unidirectional process from the professional to the stroke survivor who enacted this professionally driven advice, and it mostly concerned practical management strategies whereby something physical was being done to the body:

Timed phases in the day where left hand must be in use, limited to 5 minutes; use of robotic arm once a week during physio session; additional massaging and sensory exercises daily;[...] mirror box to try to activate nerves; TENS machine on triceps and back of wrist [..] In the early stages when trying to wake up the arm it is very much a case of throw everything at it a[nd] see what works. Structured plan was a lot further down the path once movement had started.

Survey respondent, female, age 30-39 years, 4 months post-stroke, ABILHAND score: 20

I would say just be patient and do everything they tell you to do in terms of stretching, medication and exercises and try to use the hand even though it's difficult [...] even maybe if it's just holding something, you know, so it's not just there and, you know, so it's not dead. So just try, [...] and you'd be surprised there's some things you can do with it.

Interviewee, male, age 30-39 years, 11 months post-stroke, upper limb recovery: minimal

Investing in recovery

This sub-theme describes stroke survivors' experiences of negative and/or positive emotions in response to the recovery process; and the subsequent impact on stroke survivors investing in recovery. 
Stroke survivors described feelings of anger, frustration, worry and sadness at the loss of the arm or lack of improvement, and much of this related to coming to terms with the consequences of stroke.

Well, I've come to terms with it, put it that way, I would love to be, I can sit and think of all the things I'd love to do but I need two hands for it and I don't have that anymore so I just accept it really, but one thing is my husband is very good to me, he helps me. He's always the other hand.

Interviewee, female, age 80-89 years, 5 years post stroke, upper limb recovery: none But participants also experienced positive feelings of excitement, joy, pride and selfconfidence when progress was being made; whereby progress could constitute improvement of the affected arm, discovering ways of carrying out activities differently, and/or becoming at ease with the way stroke had affected the arm. After [the stroke], I thought my hand is going to be like this [limp] forever. I was very very worried, very frightened, you know. [...] Because I'm a right-handed man, and I started eating through my left and everything, so I was very very worried. [...] So it was very very difficult, but l'm good now, it's getting better.

Interviewee, male, age 40-49 years, 3 years post-stroke, upper limb recovery: fair I'm doing wonderful actually, I get on really well. [...] To start with, I was absolutely desperate; I can't explain how desperate I was for that arm to work. I tried and I tried and tried, but now I just get on with it. I just have to sort of focus and think, right, there's people worse off than I am, there's people in comas and there's me. [...] I don't give in, I do the best I can every day. I go shopping, I do, I cook, yes I do, I do really well.

Interviewee, female, age 50-59 years, 8 years post-stroke, upper limb recovery: none The contrast and interplay between negative and positive emotional responses was exemplified in the incremental nature of recovery, in which small improvements gave 
rise to encouragement, and not experiencing any progress could lead to frustration or sadness:

Well I think it's to keep people's optimism up and, you know, to give them the hope they can improve things. And so sometimes it can get you down a little bit, you know, things don't seem to move much, even little things can mean quite a lot. So, you know, even little changes, and if you know they're going in the right direction and that there's probably even greater chance later on of further improvement then, you know, that to me would seem to be a good sign.

Interviewee, male, age 70-79 years, 6 months post-stroke, upper limb recovery: fair

This guided how invested stroke survivors were with respect to working on the affected arm. Individuals navigated various paths, from concentrating on exercising and using the affected arm as much as possible to using the non-affected arm to fill in or take over. One interviewee, for example, described how some ability to move his affected hand had returned in hospital while he underwent mirror box therapy. Initially keen to continue with his daily programme at home, he constructed his own mirror box. But he eventually became frustrated at the lack of further improvement and decided to practice writing with his unaffected hand:

To be honest, I haven't had much success with the mirror box at the moment, I find that quite frustrating because I've been doing it for a long time, I've been doing it since I was in hospital. [...] I'm currently learning to be right handed to write, I was left handed, yeah, but I've figured out that basically it'll be easier to learn to write again right handed than it will to wait for my [left hand].

Interviewee, male, age 40-49 years, 8 months post-stroke, upper limb recovery: minimal 


\section{Choosing a positive mind-set}

This sub-theme describes stroke survivors deliberately adopting a positive mind-set, and also recommending to others the importance of keeping a positive and hopeful outlook. Stroke survivors described the overall mind-set they considered helpful with respect to managing the severely impaired arm, and this encompassed a positive and hopeful outlook and an attitude of resilience and determination. Participants said that it was important to 'keep at it', 'even just' massaging/stretching the arm, doing a very basic exercise or small movement, and not to give up on the arm. Importantly, this represented a conscious attitude stroke survivors chose to assume, rather than a general, pervading state of positivity. Also when individuals experienced feelings of sadness, frustration or anger at the loss of their arm or lack of improvement, this was the mind-set they deliberately adopted and considered most helpful, and they would also recommend to others who are in a similar situation. It was also emphasised that 'it's a long process', 'there are no shortcuts', 'think years, not weeks and months', 'you have to put the work in', and 'look at it as a challenge':

It is better than it was. If I look after it, it will get better. It never changed in the hospital, now I can feel if someone touches [it]. Everything it does, it needs help. It's slow, but it is trying. I do not get angry with it. It is a bit heavy, but it is trying. Sometimes it does not obey me, or does not do what I want. Sometimes I don't know what is happening in there. [...] Look after it. Do not give up, even if it does not do anything, keep talking to it. Make sure it is not in the wrong place and keep it clean. Work on it several times a day.

Survey respondent, female, age 60-69 years, 6 months post-stroke, ABILHAND score: 18

I talk, I know it sounds strange, but I talk to the hand to say I love it and that it's mine, and it's still with me, and I try to use it in everything I do in everyday life. 
Interviewee, female, age 60-69 years, 4 years post-stroke, upper limb recovery: good

Researcher: So, if I could ask you as the next question, what advice would you give somebody in your position, somebody whose arm and hand have been affected like yours, what would you say to somebody as a piece of advice or a tip or...

F1: Don't give up.

M6 [dysphasic speaker]: Yes, yes, yes!

F2 [dysphasic speaker]: Strong, yeah, life.

M1: Yeah.

Excerpt from focus group transcript; $\mathrm{F} 1$ - female, age $30-39$ years, 4 years poststroke, upper limb recovery: fair; F2 - female, age 50-59 years, 12 years poststroke, upper limb recovery: minimal; M1 - male, age $40-49$ years, 6 years poststroke, upper limb recovery: fair; M6 - male, age 40-49 years, 4 years poststroke, upper limb recovery: minimal

Several stroke survivors talked about how rehabilitation professionals had predicted their arm may never improve. They said this message had a strong negative impact on them, and professionals should not make such predictions. Importantly, participants did not seek the promise of improvement or recovery. Rather, they emphasised the importance of maintaining hope in order to strengthen resilience and determination, and bolster an attitude of keeping going and not giving up:

I think the physio didn't bother with me because I was walking, and I said to the occupational therapist, what will I do with my arm, there's no exercises to do my arm. And she said, well, it may not get better, basically, don't bother. And I was so upset.

Focus group participant, female, age 30-39 years, 4 years post-stroke, upper limb recovery: fair 
[Dysphasic speaker:] I was very down, really down because [pause] the doctors said to me, when I had the stroke, give it two years and when the two years is over that's as good as it's gonna be. And two years later, I was really, I thought, yeah, that's it, that's me, that's all, it's me, do you know what I mean, so I used to lock myself away. [...] Doctors to give me two years - No, it's on and on and on and on and on. It's wrong. Wrong. Yeah. Yeah. Keep working it, keep working it, keep working it.

Interviewee, male, age 50-59 years, 15 years post-stroke, upper limb recovery: minimal

There are no shortcuts - it's about repetition, intensity and duration. Trust that over a long time efforts will be rewarded. The pace of change is immeasurable over weeks \& months. Look at changes over years.

Survey respondent, male, age 40-49 years, 3 years post-stroke, ABILHAND score: 24

Be very patient, improvements may not happen immediately. Don't be frightened to talk to people about your disability if they are interested and want to know or understand. Do not be embarrassed. Stay positive and believe in yourself.

Survey respondent, female, age 60-69 years, 9 months post-stroke, ABILHAND score: 16

Nobody knows what's going to happen tomorrow, we just wish for the best, that's all I can tell that person, hope for the best. If it works, fine, if it doesn't, have hope. [...] It's your hand, there's nothing you can do, it's there, it has happened, so just be positive and just keep going. [...] Because you can't promise it's going to get better or it's not going to get better, nobody knows. You hope for the best, though. Interviewee, male, age 40-49 years, 3 years post-stroke, upper-limb recovery: minimal 


\section{Choosing and expanding}

This overarching theme represents personal agency and resourcefulness of stroke survivors. It describes the person choosing management strategies that work for them, and expanding on professional advice through modifying, discovering, trying out and adding new strategies. Much of this is driven by self-discovery and through connection and exchange with other stroke survivors.

Stroke survivors' overall experience of managing the severely impaired arm was characterised by choosing from and expanding on professionals' advice and guidance. Stroke survivors tried out strategies, chose what worked for them and what to carry on with, while leaving aside what they found did not work for them. They expanded on professionals' advice by modifying, adapting, discovering, trying out and adding new management strategies and avenues:

I was given some exercises to do by my physiotherapist, just using a weight, really. It was lifting my arm up and lowering it down. But I've personally expanded that exercise to include across my body, wide, up and, you know, all round.

Interviewee, male, age 50-59 years, 7 months post-stroke, upper limb recovery: fair

This represented self-management principles of individual agency and resourcefulness in action, developing personally meaningful self-management from initial standard rehabilitation practice. An illustrative example was a survey respondent's home-made adaptation for attaching a guitar pick to his non-functional hand, which allowed him to strum the strings and continue his lifelong hobby of playing the guitar. Another illustrative example was one participant's account of how her fingers tended to fold, and her arm would bend and pull tight against her body. The participant found that 
massage improved tightness and heaviness of the affected arm, allowing a more neutral position, which supported her self-image and confidence to appear in public.

Choosing and expanding was driven mainly by self-discovery, often with the support of family members and friends, and by connecting, sharing and exchanging with other stroke survivors, while navigating one's emotional responses and trying to maintain a positive mind-set:

\begin{abstract}
Quite a lot of useful information from Facebook, [...] a lot of the stroke survivors will post on there their experiences and things that they've tried and have worked, which is a lot of positive stuff on there. [...] just trying to basically listen to success stories on there, and just trying to involve my arm a lot more.

Interviewee, male, age 40-49 years, 8 months post-stroke, upper limb recovery: minimal
\end{abstract}

I find being in a group and talking about your experiences is a very good way, because everyone has their opinions, and you gain benefits from what some people say, or some things that you may not have thought of that help you. [...] Sometimes, as they say, a problem shared is a problem halved, so that way you feel 'well, I'm not the only one going through this, there's other people like me'.

Interviewee, female, age 50-59 years, 2 years post-stroke, upper limb recovery: good

In addition, stroke survivors prioritised their time, weighing up the relative importance and perceived benefit of management strategies in the context of their lives:

I could do exercises, the time I wake up in the morning to the time I go to bed, but you've got a life to lead.

Focus group participant, male, age $40-49$ years, 6 years post-stroke, upper limb recovery: fair 


\section{Views on a self-management intervention for the severely impaired arm}

From their own experience, study participants could appreciate the value of a selfmanagement intervention that would capture and pass on their experience-based knowledge to other stroke survivors:

Something about their experience of having a stroke, and how it made them feel, or how difficult it was, you know. And we'll all have differences but we all share something similar. [...] So I find something like that is good.

Interviewee, female, age 50-59 years, 2 years post-stroke, upper limb recovery: good

Several participants commented that early rehabilitation often focused on the lower limb and mobility, and suggested that a self-management intervention specific to the upper limb could complement this.

I think what they were just concentrating on was my legs, not my hand. [...] I did some therapy at the hospital, when I was in the stroke unit, but then it wasn't for the hand, it wasn't as much as on the leg. [...] Yes, if I'd known, well, I think while they were concentrating on my leg, I would have concentrated on my hand too, my right hand too.

Interviewee, female, age 40-49 years, 13 years post-stroke, upper limb recovery: none The content of such an intervention would need to be sensitive to the distinction between individuals who can move their affected arm at least a little and therefore have 'something to work with'; as opposed to those who have 'nothing', i.e. are unable to elicit any volitional movement. Participants preferred a self-management intervention to be presented by a stroke survivor, as this was considered to support authenticity, credibility, and motivation for behaviour change:

Health professionals can tell you but they haven't had experience. They've seen the experience but they haven't had experience, and 
you understand it better when somebody has got experience, telling you, "this is how I feel, this is how hard it was, this is how, what can be done". [...] You try to take that on board more when somebody tells you their experience.

Interviewee, male, age 40-49 years, 3 years post-stroke, upper-limb recovery: minimal

Suggested formats and modes of delivery varied widely amongst participants and included written and audio-visual resources, digital and social media applications, interactive group-based events, and formal presentations. These represent different learning styles and personal preferences.

\section{Discussion}

This study has provided insights into stroke survivors' experiences of managing the severely affected upper limb; and views on a self-management intervention specifically for the severely impaired arm. To the authors' knowledge, this is the first study to explore this topic in stroke survivors in the UK, and against a theoretical background of self-management.

Describing a novel theme of choosing and expanding, this study has brought to the fore facets of stroke survivors' lived experience that are reflective of stroke selfmanagement. Self-management interventions build on aspects relating to the individual, e.g. personal self-efficacy, problem-solving skills, and ability to seek out and access resources $[27,28]$; and collective/social processes such as vicarious learning, social comparison, and navigating and negotiating within social networks $[29,30]$. This study has provided many examples of these mechanisms in action (i.e. self-discovery and connecting and exchanging with other stroke survivors), thus confirming these 
processes through stroke survivors' experience-based accounts, and also demonstrating how these may support personal resilience and psychosocial wellbeing. This indicates the potential benefits of a self-management intervention in this context, particularly with a view to supporting long-term care, as all study participants had had a non-functional upper limb at 3 months post stroke, a time when formal stroke rehabilitation services will often reduce or discharge and self-management becomes increasingly important.

Generic stroke self-management interventions have demonstrated positive effects on quality of life and self-efficacy [11], and topical reviews and clinical stroke guidelines increasingly call for self-management to be incorporated in stroke rehabilitation, especially as a strategy for supporting long-term care $[23,24,25,26]$. Several study participants highlighted that rehabilitation may prioritise lower limb and mobility goals over the upper limb, and this has also been described in previous research [31]. There is therefore scope for a self-management intervention that specifically addresses the severely impaired arm to be incorporated into formal stroke rehabilitation. Although the Stroke Recovery and Rehabilitation Roundtable taskforce [17] have recently published a call for more ambitious research (aiming at breakthrough treatments for restitution and brain repair, rather than pragmatic interventions that could be delivered in existing healthcare settings), there nevertheless remains an evidence gap regarding practical guidance for formal rehabilitation and sustainable and meaningful long-term management strategies for stroke survivors. It may be possible to draw a parallel with the field of dementia research, in which the search for curative treatments continues, while holistic psychosocial interventions have had positive impact for people with dementia and their caregivers over the past two decades [33]. 
Similarly, stroke survivors with severe upper limb impairment could benefit from a tailored self-management intervention, until restorative treatments have been developed and made widely available.

Findings from the present study give original and useful insights to inform the design of such an intervention. At a theoretical level, the overarching theme choosing and expanding and sub-themes investing in recovery and choosing a positive mind-set are particularly pertinent. Conceptually, these themes align self-management of the severely affected upper limb, firstly with 'self-help' in the sense of a 'natural' human activity [B]; and secondly with a 'broader' approach to self-management, aiming to support people to manage well with their condition and taking into account what matters most to them (as opposed to a 'narrow' approach, aiming to control the condition) [C]. Importantly, this presents a complementary, yet alternative view to the current mainstream of research in upper limb rehabilitation post stroke, which focuses largely on novel technology-based interventions (e.g. robotics, neurophysiological stimulation, interactive technologies), maximising therapy intensity, and a predictionbased model of rehabilitation practice $[7,17,18,19]$.

While these mainstream approaches may lead to some advancement, there are also inherent limitations, relating to both research methodology and implementation in practice. Rehabilitation trials of technology-based interventions and high-intensity task practice are often highly selective in their eligibility criteria, leading to downstream problems of generalisability and limited relevance for large patient groups. Highintensity therapy also presents implementation challenges of participant burden, practicality and sustainability. The Predict Recovery Potential algorithm has recently been shown to hold some clinical utility in directing acute and subacute rehabilitation 
resources [19], but this evidence is currently limited to the first 6 months after stroke and more applicable to individuals with mild to moderate upper limb impairment. Moreover, as reflected in this study's theme of choosing a positive mind-set, there is a need to consider the long-term psychosocial impact of negative predictions on stroke survivors and their surrounding network of family and friends; and the multiplicity of human life, in which unexpected and unpredictable developments can bring positive outcomes. These sentiments are echoed in the qualitative study by Barker and Brauer, which described key themes of 'keeping the door open' and 'hanging in there', concluding that there is a need to develop training strategies that match needs and aspirations of stroke survivors and that place no time limits on recovery [9]. Furthermore, syntheses of qualitative research demonstrate that maintaining hope is often a preference of stroke survivors themselves [20,21], and quantitative studies have provided evidence that hope is positively associated with rehabilitation outcomes $[9,22]$.

In addition to conceptual considerations, findings from this study also provide more factual information on how stroke survivors may envisage the format and mode of delivery of a self-management intervention for the severely affected arm. Unsurprisingly, suggestions for the design of an intervention and resources varied widely, presenting the challenge of balancing widespread appeal and utility against accommodating individual learning styles and preferences. There was, however, a uniform view of study participants that a self-management intervention should be presented, or at least co-presented, by a stroke survivor, as opposed to a rehabilitation or research professional. This confirms that connecting with people who experience a similar situation can constitute a powerful aspect of self-management interventions. 
The question whether specific treatments or management strategies for the strokeaffected arm were supported by research evidence was notably absent from the data. This may seem a surprising finding in the UK, where public healthcare is underpinned by a strong ethos of evidence-based practice. It may indicate a tendency of stroke survivors to not explicitly recognise the purpose of research evidence in the selection and use of rehabilitation interventions; or to not explicitly consider the relevance of research evidence in relation to their own personal circumstances. Rehabilitation and research professionals may be best placed to give an understanding of evidence-based practice principles and contribute this type of content to a self-management intervention.

\section{Study limitations}

Limitations to this study relate to characteristics of qualitative research, which trade off the representativeness of large samples against in-depth insights. Study participants were a self-selected group of stroke survivors who chose to engage with the research. Nevertheless, these empirical findings can address and further a wide range of issues regarding the impact of stroke on individuals and the delivery of services [32]. While recruitment to interviews and the focus group exceeded the target, survey responses were below the initial estimate. Importantly, this does not limit the validity of survey data. Rather, the survey should be understood as a qualitative data collection strategy for increasing inclusiveness, allowing 20 more stroke survivors to share their views and experiences, who otherwise might not have taken part in the research. Inclusiveness was further supported by enabling participants with restricted mobility to participate from their home, and using an 
interview style that supports people with dysphasia in communicating, all of which contributed to diversity in the sample. In a qualitative methodology this increases the representativeness of findings; however, it is acknowledged that these data may reflect cultural and attitudinal characteristics particular to the UK context. One study strength was the use of mixed data in the survey, where respondents' free text answers confirmed and complemented qualitative data from interviews and the focus group, and quantitative data provided descriptions of respondents' upper limb impairment and function. Another strength was the involvement of study advisors who themselves were stroke survivors with personal experience of severe upper limb impairment. While interview transcripts or summaries of accounts were not returned to participants for verification, triangulation of data sources and study advisors' peer review of the data analysis support the credibility of findings.

\section{Implications for practice and future research}

Findings from this study may inform rehabilitation practice to enhance the provision of self-management support to stroke survivors with severe upper limb impairment. For example, rehabilitation professionals may actively support stroke survivors in choosing from, and expanding on professional advice and guidance; and remain mindful that a positive and hopeful outlook is often important to many stroke survivors. Future research may add to this study by purposively exploring experiences of groups that were not represented in this sample, for example carers who are very involved in the management of the stroke survivor's affected upper limb; or stroke survivors who may have alternative experiences and different views to the participants in this study. Future research may also build on this study, by developing and evaluating a stroke 
self-management intervention specifically for the severely affected arm. Participatory co-design methods are best suited to co-produce such an intervention, to enable stroke survivors' experiences and voices to feature in an authentic and credible manner [27].

\section{Conclusions}

In conclusion, this study explored a knowledge gap with respect to stroke survivors' experiences of, and management strategies for, the severely impaired upper limb. The findings show how stroke survivors choose and expand professionally driven strategies, developing personally meaningful management from initial standard rehabilitation practice. Stroke survivors' accounts reflect and resonate with principles of self-management, demonstrating scope for a tailored self-management intervention and its potential benefits. These findings extend the current evidence base and may be used to inform rehabilitation practice, and the development of a stroke selfmanagement intervention specifically addressing the severely affected upper limb. 


\section{References}

[1] Sentinel Stroke National Audit Programme. Mind the Gap! The Third SSNAP Annual Report. London: Royal College of Physicians; 2016.

[2] Lee S, Shafe ACE, Cowie MR. UK Stroke Incidence, Mortality and Cardiovascular Risk Management 1999-2008: Time-Trend Analysis from the General Practice Research Database. BMJ Open. 2011;1:e000269.

[3] Lawrence ES, Coshall C, Dundas R, Steward J, Rudd AG, Howard R, et al. Estimates of the Prevalence of Acute Stroke Impairments and Disability in a Multiethnic Population. Stroke 2001;32:1279-1284.

[4] Kwakkel G, Kollen BJ, Van der Grond J, Prevo AJ. Probability of Regaining Dexterity in the Flaccid Upper Limb: Impact of Severity of Paresis and Time Since Onset in Acute Stroke. Stroke. 2003;34(9):2181-2186.

[5] Lai SM, Studenski S, Duncan PW, Perera S. Persisting Consequences of Stroke Measured by the Stroke Impact Scale. Stroke. 2002;33:1840-1844.

[6] Pollock A, St George B, Fenton M, Firkins L. Top 10 Research Priorities Relating to Life After Stroke. Consensus from Stroke Survivors, Caregivers, and Health Professionals. Int J Stroke. 2014;9:313-320.

[7] Pollock A, Farmer SE, Brady MC, Langhorne P, Mead GE, Mehrholz J, et al. Interventions for Improving Upper Limb Function after Stroke. Cochrane Database Syst Rev. 2014;11:CD010820.

[8] Ch'ng AM, French D, McLean N. Coping with the Challenges of Recovery from Stroke. J Health Psychol. 2008;13:1136-1146. 
[9] Barker RN, Brauer SG. Upper Limb Recovery After Stroke: The Stroke Survivors' Perspective. Disabil Rehabil. 2005;27:1213-1223.

[10] Barker RN, Gill TJ, Brauer SG. Factors Contributing to Upper Limb Recovery After Stroke: A Survey of Stroke Survivors in Queensland Australia. Disabil Rehabil. 2007;29:981-989.

[11] Fryer CE, Luker JA, McDonnell MN, Hillier SL. Self Management Programmes for Quality of Life in People with Stroke. Cochrane Database Syst Rev. 2016;8:CD010442.

[A] Barlow J, Sturt J, Hearnshaw H. Self-management interventions for people with chronic conditions in primary care: examples from arthritis, asthma and diabetes. Health Educ J. 2002;61:365-378.

[12] Fetters MD, Curry LA, Creswell JW. Achieving integration in mixed methods designs - principles and practices. Health Serv Res. 2013;48:2134-2156.

[13] Penta M, Tesio L, Arnould C, Zancan A, Thonnard J-L. The ABILHAND Questionnaire as a Measure of Manual Ability in Chronic Stroke Patients: Rasch-Based Validation and Relationship to Upper Limb Impairment. Stroke. 2001;32:16271634.

[14] Ashford S, Slade M, Nair A, Turner-Stokes L. Arm Activity Measure (ArmA) Application for Recording Functional Gain Following Focal Spasticity Treatment. Int J Ther Rehabil. 2015;21:10-17.

[15] Spencer L, Ritchie J, Ormston R, O'Connor W, Barnard M. Analysis: Principles and Processes. In: Ritchie J, Lewis J, McNaughton Nicholls C, Ormston R (Eds.). Qualitative Research Practice. 2nd Ed. London: Sage;2014. 
[16] O'Brien B, Harris IB, Beckman TJ, Reed DA, Cook DA. Standards for reporting qualitative research: A synthesis of recommendations. Acad Med. 2014;89:1245-1251.

[17] Bernhardt J, Hayward KS, Kwakkel G, Ward NS, Wolf SL, Borschmann K, et al. Agreed Definitions and a Shared Vision for New Standards in Stroke Recovery Research: The Stroke Recovery and Rehabilitation Roundtable Taskforce. Int J Stroke. 2017;12:444-450.

[18] Hayward KS, Schmidt J, Lohse KR, Peters S, Bernhardt J, Lannin NA, Boyd LA. Are We Armed with the Right Data? Pooled Individual Data Review of Biomarkers in People with Severe Upper Limb Impairment after Stroke. Neuroimage Clin. 2017; 15:53-55.

[19] Stinear CM, Byblow WD, Ackerley SJ, Barber PA, Smith M-C. Predicting Recovery Potential for Individual Stroke Patients Increases Rehabilitation Efficiency. Stroke. 2017;48:1011-1019.

[20] Pearce G, Pinnock H, Epiphaniou E, Parke HL, Heavey E, Griffiths CJ, et al. Experiences of Self-Management Support Following a Stroke: A Meta-Review of Qualitative Systematic Reviews. PLoS ONE. 2015;10:e0141803.

[21] Bright FA, Kayes NM, McCann CM, McPherson KM. Understanding Hope After Stroke: A Systematic Review of the Literature Using Concept Analysis. Top Stroke Rehabil. 2011;18:490-508.

[22] Kortee KB, Stevenson JE, Hosey MM, Castillo R, Wegener ST. Hope Predicts Positive Functional Role Outcomes in Acute Rehabilitation Populations. Rehabil Psychol. 2012;57:248-255. 
[23] Dobkin BH. Behavioral Self-Management Strategies for Practice and Exercise Should be Included in Neurologic Rehabilitation Trials and Care. Curr Opin Neurol. 2016;29:693-699.

[24] Intercollegiate Stroke Working Party. National Clinical Guideline for Stroke. $5^{\text {th }}$ Ed. London: Royal College of Physicians;2016.

[25] Mayo NE. Stroke Rehabilitation at Home. Lessons Learned and Ways Forward. Stroke. 2016;47:1685-1691.

[26] Winstein CJ, Stein J, Arena R, Bates B, Cherney LR, Cramer SC, et al. Guidelines for Adult Stroke Rehabilitation and Recovery. A Guideline for Healthcare Professionals From the American Heart Association/American Stroke Association. Stroke. 2016;47:e98-e169.

[27] Jones F, Pöstges H, Brimicombe L. Building Bridges Between Healthcare Professionals, Patients and Families: A Coproduced and Integrated Approach to Self-Management Support in Stroke. NeuroRehabilitation, 2016;39: 471-480.

[28] Lorig K, Holman HR. Self-Management Education: History, Definition, Outcomes and Mechanisms. Ann Behav Med. 2003;26: 1-7.

[29] Sadler E, Sarre S, Tinker A, Bhalla A, McKevitt C. Developing a Novel Peer Support Intervention to Promote Resilience after Stroke. Health Soc Care Community. 2016;25:1590-1600.

[30] Vassilev I, Rogers A, Kennedy A, Koetsenruijter J. The Influence of Social Networks on Self-Management Support: A Metasynthesis. BMC Public Health. 2014;14:719.

[31] West T, Bernhardt J. Physical Activity in Hospitalised Stroke Patients. Stroke Res Treat. 2012;2012:813765. 
[32] McKevitt C, Redfern J, Mold F, Wolfe C. Qualitative Studies of Stroke. A Systematic Review. Stroke. 2004;35:1499-1505.

[33] Moniz-Cook E, Vernooij-Dassen M, Woods B, Orrell M, Interdem Network.

Psychosocial Interventions in Dementia Care Research: the INTERDEM Manifesto. Aging Ment Health. 2011;15:283-290.

[B] Kendall E, Rogers A. Extinguishing the social?: state sponsored self-care policy and the Chronic Disease Self-management Programme. Disabil Soc. 2007;22:129143.

[C] Morgan HM, Entwistle VA, Cribb A, Christmas S, Owens J, Skea ZC, Watt IS. We need to talk about purpose: a critical interpretive synthesis of health and social care professionals' approaches to self-management support for people with long-term conditions. Health Expect. 2017;20:243-259. 
Figure 1. Overview of themes (analytic framework)

\section{Choosing and expanding}

Stroke survivors demonstrate personal agency and resourcefulness. They choose management strategies that work for them, and expand on professional advice through modifying, adapting, discovering, trying out and adding new strategies. Much of this is driven by self-discovery, and through connection and exchange with other stroke survivors.

\section{Enacting professionally driven advice}

Rehabilitation professionals make recommendations and give advice and guidance, especially during the first weeks and months following stroke. This is then enacted by stroke survivors in a unidirectional process.

\section{Choosing a positive mind-set}

Stroke survivors deliberately seek to adopt a positive mind-set, and also recommend to others the importance

of keeping a positive and hopeful outlook.

\section{Investing in recovery}

Stroke survivors experience negative and/or positive emotions in response to the recovery process. These subsequently influence how invested stroke survivors are in working on their affected arm.

Table 1. Participant characteristics

\begin{tabular}{|l|l|l|l|l|}
\hline & $\begin{array}{l}\text { Interview } \\
\text { participants } \\
(\mathrm{N}=16)\end{array}$ & $\begin{array}{l}\text { Focus group } \\
\text { participants } \\
(\mathrm{N}=6)\end{array}$ & $\begin{array}{l}\text { Survey } \\
\text { respondents } \\
(\mathrm{N}=20)\end{array}$ & $\begin{array}{l}\text { Combined } \\
\text { sample } \\
(\mathrm{N}=42)\end{array}$ \\
\hline Age group & - & - & - & - \\
\hline$<30$ years & 5 & 4 & 7 & $16(38.1 \%)$ \\
\hline $30-49$ years & 8 & 2 & 9 & $19(45.2 \%)$ \\
\hline $50-69$ years & 3 & - & 4 & $7(16.7 \%)$ \\
\hline$\geq 70$ years & & & 8 & $16(38.1 \%)$ \\
\hline Sex & 6 & 2 & 12 & $26(61.9 \%)$ \\
\hline Female & 10 & 4 & - & $1(2.4 \%)$ \\
\hline Male & & - & 3 & $9(21.4 \%)$ \\
\hline Ethnicity & 1 & - & & \\
\hline Asian & 6 & & & \\
\hline Black & & - & & \\
\hline
\end{tabular}




\begin{tabular}{|c|c|c|c|c|}
\hline White & 9 & 6 & 17 & $32(76.2 \%)$ \\
\hline \multicolumn{5}{|l|}{$\begin{array}{l}\text { Time since stroke } \\
\text { onset }\end{array}$} \\
\hline$<6$ months & - & - & 1 & $1(2.4 \%)$ \\
\hline 6-12 months & 4 & - & 5 & $9(21.4 \%)$ \\
\hline $1-2$ years & - & - & 4 & $4(9.5 \%)$ \\
\hline $2-5$ years & 8 & 3 & 3 & $14(33.3 \%)$ \\
\hline $5-10$ years & 1 & 1 & 4 & $6(14.3 \%)$ \\
\hline$>10$ years & 3 & 2 & 3 & $8(19.0 \%)$ \\
\hline \multicolumn{5}{|l|}{ Type of stroke } \\
\hline Ischemic & 7 & 1 & 12 & $20(47.6 \%)$ \\
\hline Haemorrhagic & 8 & - & 5 & $13(31.0 \%)$ \\
\hline Not known & 1 & 5 & 3 & $9(21.4 \%)$ \\
\hline \multicolumn{5}{|l|}{$\begin{array}{l}\text { Stroke-affected } \\
\text { upper limb }\end{array}$} \\
\hline Left & 7 & - & 12 & $19(45.2 \%)$ \\
\hline Right & 9 & 6 & 8 & $23(54.8 \%)$ \\
\hline \multicolumn{5}{|l|}{$\begin{array}{l}\text { Primary } \\
\text { presentation of } \\
\text { upper limb }\end{array}$} \\
\hline Paresis/weakness & 10 & 3 & 6 & 19 (45.2\%) \\
\hline $\begin{array}{l}\text { Spasticity/ } \\
\text { hypertonicity }\end{array}$ & 6 & 3 & 14 & $23(54.8 \%)$ \\
\hline \multicolumn{5}{|l|}{$\begin{array}{l}\text { Dominant hand } \\
\text { affected }\end{array}$} \\
\hline Yes & 10 & 6 & 6 & $22(52.4 \%)$ \\
\hline No & 6 & - & 14 & 20 (47.6\%) \\
\hline \multicolumn{5}{|l|}{$\begin{array}{l}\text { Level of upper } \\
\text { limb recovery }^{\text {a }}\end{array}$} \\
\hline None & 5 & 2 & - & $n / a$ \\
\hline
\end{tabular}




\begin{tabular}{|c|c|c|c|c|}
\hline Minimal & 6 & 2 & - & $\mathrm{n} / \mathrm{a}$ \\
\hline Fair & 3 & 2 & - & $\mathrm{n} / \mathrm{a}$ \\
\hline Good & 2 & - & - & $\mathrm{n} / \mathrm{a}$ \\
\hline Complete & - & - & - & $\mathrm{n} / \mathrm{a}$ \\
\hline $\begin{array}{l}\text { Self-reported } \\
\text { measures }\end{array}$ & & & & \\
\hline $\begin{array}{l}\text { ABILHAND Manual } \\
\text { Ability Measure } \\
\text { (median, range) }^{\text {b }}\end{array}$ & - & - & $24(0,40)$ & $\mathrm{n} / \mathrm{a}$ \\
\hline $\begin{array}{l}\text { Arm Activity } \\
\text { Measure (ArmA; } \\
\text { median, range) }{ }^{c}\end{array}$ & - & - & $11(3,25)$ & $\mathrm{n} / \mathrm{a}$ \\
\hline \multicolumn{4}{|c|}{$\begin{array}{l}\text { a Investigator's assessment at the time of interview/focus group } \\
\text { (none: no volitional movement of elbow and hand; minimal: some } \\
\text { volitional movement of elbow and hand but insufficient for } \\
\text { functional activity; fair: volitional movement sufficient for functional } \\
\text { movement but too effortful for everyday use; good: volitional } \\
\text { movement sufficient for functional movement in everyday use; } \\
\text { complete: considered same as pre-stroke) } \\
\text { b Score range 0-46, higher score represents better manual ability }\end{array}$} & \\
\hline
\end{tabular}

